\title{
Detection of Small LEO Debris with Line Detection Method
}

\author{
By Toshifumi Yanagisawa and Atsushi NaKaJIMA \\ Japan Aerospace Exploration Agency, Tokyo, Japan
}

(Received February 6th, 2004)

\begin{abstract}
This paper proposes a new method known as "the line detection method" to detect small pieces of low Earth orbit (LEO) debris. A direction is assumed for the line created by small LEO debris on a CCD image and the values of pixels along that direction are accumulated to improve the signal-to-noise ratio. This method can detect LEO debris that is 30 to 40 times darker than debris that can be detected by usual methods. We tested this method by using the 35-cm telescope and back-illuminated CCD camera at the Mt. Nyukasa Astronomical Observatory. One small piece of LEO debris with a radar cross section of $0.0047 \mathrm{~m}^{2}$ was detected. By using this method, the 1-m telescope and back-illuminated wide-field camera at the Bisei Spaceguard Center (BSGC) are expected to be able to detect LEO debris with a size of a few $\mathrm{cm}$. The line detection method will be used to probe the LEO small debris environment and contribute to solving the space debris problem.
\end{abstract}

Key Words: Space Debris, Optical Observation, Detection Method

\section{Introduction}

Since Sputnik was first launched in 1957, many other satellites have been launched and the number of artificial objects in orbit continues increasing. Explosions and collisions between these objects are creating large amounts of space debris. For safety, this debris must be detected and cataloged. Systems for observing space debris have been established in the USA and Russia. ${ }^{1)}$ There are believed to be some 4 million pieces of space debris larger than $1 \mathrm{~mm}$, but fewer than 10 thousand have been cataloged. ${ }^{2}$

In Japan, the Japan Space Forum is building an optical observatory at Bisei Town and a radar site at Kamisaibara Village in Okayama Prefecture. ${ }^{3)}$ The National Institute of Information and Communications Technology is studying optical observation of geostationary (GEO) debris. ${ }^{4-6)}$ Our Japan Aerospace Exploration Agency (JAXA) is investigating shape and motion determination for LEO satellites and LEO debris and developing detection technologies for dark GEO and LEO debris. ${ }^{7,8)}$

There are dense regions of LEO debris at heights of around 800 to $1,000 \mathrm{~km}$ and $1,500 \mathrm{~km}$. The amount of LEO debris in these regions continues increasing because polar orbit satellites are usually placed here and their lifetimes are a few thousand years. In addition, explosion of boosters with unused fuel has produced a great deal of debris. It is thought that these factors have produced the critical density required for a chain reaction in which debris collisions produce even more debris. We must take measures such as active orbital transfers and retrievals to deal with the situation.

We need to investigate the debris environment at heights of around 300 to $500 \mathrm{~km}$ in detail because the International Space Station (ISS) operates in this region. The current ISS

(C) 2005 The Japan Society for Aeronautical and Space Sciences debris bumper can protect each component against debris of less than $1 \mathrm{~cm}$. The orbits of debris larger than $10 \mathrm{~cm}$ are determined by observation and the ISS can move its orbit to avoid colliding with such debris. Since we have no protection against debris of 1 to $10 \mathrm{~cm}$ at present, we urgently need to improve our observation and protection abilities. Although observation of LEO debris is usually done by radar, we show that optical observation is also useful.

Techniques to separate weak signals from noise can detect small debris that has not been previously detected, and can contribute to solving the space debris problem. ${ }^{9)}$ JAXA established an optical observation facility on Mt. Nyukasa in Nagano Prefecture in January 2001 in order to develop technologies for automatic detection of unknown debris, as well as other observation techniques. ${ }^{10,11)}$ In particular, we are developing image processing to detect small debris using CCD images from the $35-\mathrm{cm}$ optical telescope at the facility.

The line detection method has been developed to detect LEO debris. It can detect the faint lines left by LEO debris on CCD images. In this method, a direction is assumed for the line and the pixel values along that direction are integrated to improve the signal-to-noise ratio and detect a faint line that is almost invisible on the images. This method enables detection of previously undetected small LEO debris and is being patented.

The line detection method is described in detail in Section 2. We observed LEO debris to evaluate the usefulness of the method. Twelve lines were detected by analyzing $20 \mathrm{CCD}$ images. We compared these lines with the catalog and confirmed that one corresponded to known debris with a size of about $7 \mathrm{~cm}$. Details of the observation and analysis are shown in Sections 3 and 4, respectively. Section 5 shows the process of comparing detected lines with the catalog and consider the confirmed known debris. The method's de- 


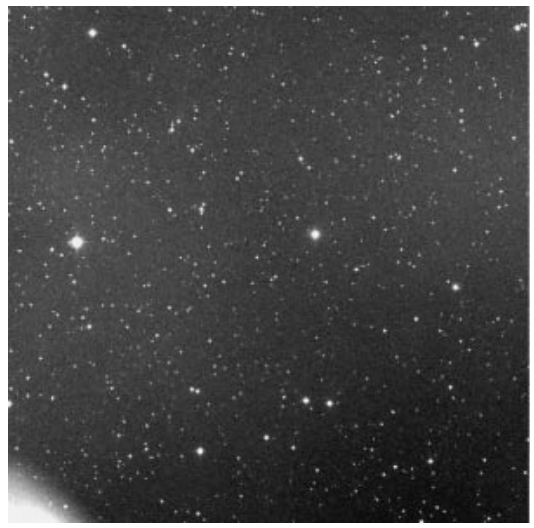

Fig. 1(a). Raw CCD image with many fixed stars. Streaks created by LEO debris are not visible on this image.

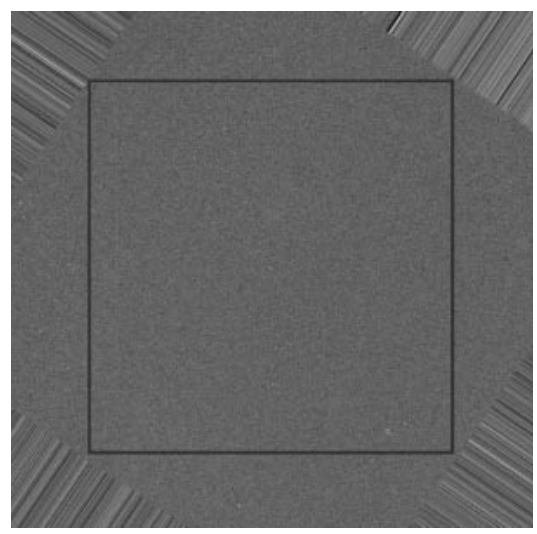

Fig. 1(c). Appropriately rotated image of Fig. 1(b).

The four corners of the image are not useful because of the rotation.

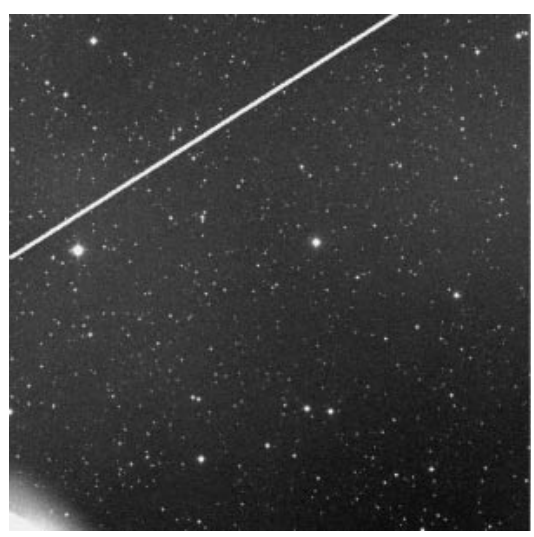

Fig. 1(e). Line detected in image.

tection abilities are investigated in Section 6. We also investigated the power of the method when used with the 1-m telescope and large CCD camera at the BSGC.

\section{Line Detection Method}

LEO satellites and LEO debris leave streaks on a CCD image when they pass through the CCD camera field of view. If they are dark, the streaks are not recognized but a signal is present although it is obscured by the sky background noise. We have developed a line detection image-

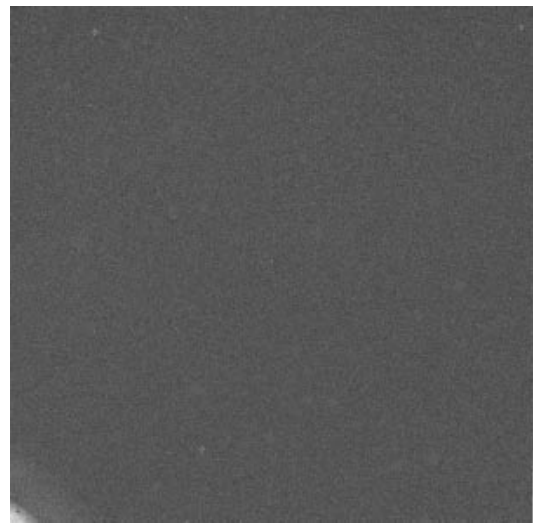

Fig. 1(b). Image masking fixed stars in Fig. 1(a). The effect of fixed stars is almost removed.

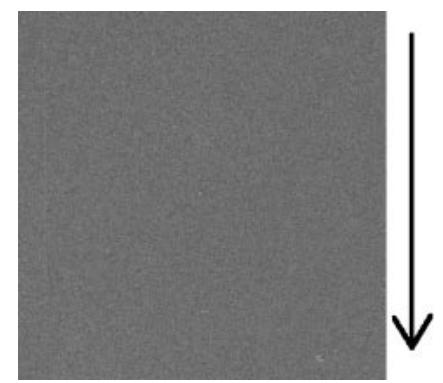

Fig. 1(d). Region that is usable after rotation extracted from raw image. Median values of all pixels of every row along the arrow are calculated.

processing technique to extract streaks created by meteors, LEO satellites, and LEO debris. Figure 1 shows the line detection method; Fig. 1(a) is a raw CCD image and the white spots are fixed stars. No lines can be observed at first sight. First, fixed stars are removed by subtracting a sequent image (Fig. 1(b)). The image is then rotated by some angle and the effective region with no impact from the rotation process is cut out (Fig. 1(c)). Finally, median values of all pixels of every row along the arrow are calculated (Fig. 1(d)). In short, one dimension of a 2-D image is reduced in order to increase the signal-to-noise ratio, and then high values are searched 
on the 1-D graph. The median is the central value of the set of values. In contrast to the mean, the median reduces any effects from unexpectedly high signals like cosmic rays, thermal noise and fixed stars. The calculated median value of every row indicates the central value of all pixels of the row that means numbers of pixels belonging to the row show around the value. A higher median value for one row than for other rows means that the row as a whole shows a higher value than other rows. This indicates that a line has been detected along the row. Calculating median values reduces the sky background noise efficiently as follows:

$$
\sigma_{\text {median }}=\frac{1.2}{\sqrt{N}} \sigma_{\text {sky }}
$$

Here, $\sigma_{\text {sky }}$ and $\sigma_{\text {median }}$ are the sky background noise and the noise level after calculating median values, respectively. $N$ is the number of pixels along one direction. The factor 1.2 was calculated by Monte Carlo simulations. ${ }^{12)}$ Reducing the sky background noise means dark lines that are invisible in the noise can be detected. By using a CCD camera with $N$ pixels in one row, the method can detect $\sqrt{N} / 1.2$ times darker lines than the normal detection of the camera. The last two parts of the process (Figs. 1(c) and 1(d)) are repeated for every angle to detect faint lines.

Figure 2 shows an example of a line detected in an image using the line detection method. The value of each pixel in analog-to-digital units (ADU) is plotted against the pixel numbers perpendicular to one direction. Constants were added to the upper two plots for clear presentation. The bottom plot shows the noise level of the raw CCD image. The middle plot shows the median values of every 20 pixels along the same direction. A line effect is visible at the center. The uppermost plot shows the median values of all pixels along that direction. The effect is clear and appears only when the line direction corresponds exactly with the rotation angle. From the rotation angle and the coordinate of the effect, the actual position of the detected line in the image is determined, as shown in Fig. 1(e).

There are several thousand pixels along any one line in recent CCD cameras. Equation (1) shows that this method can

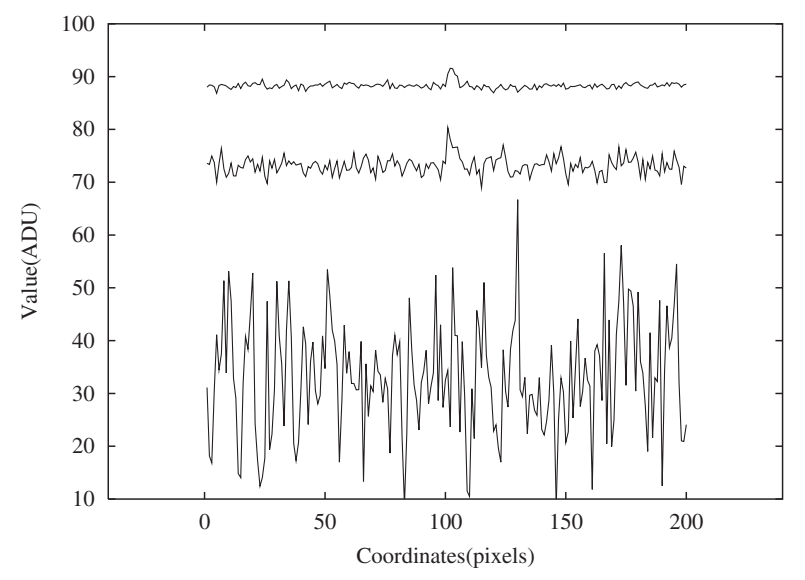

Fig. 2. Example of line effect detected in image using line detection method.

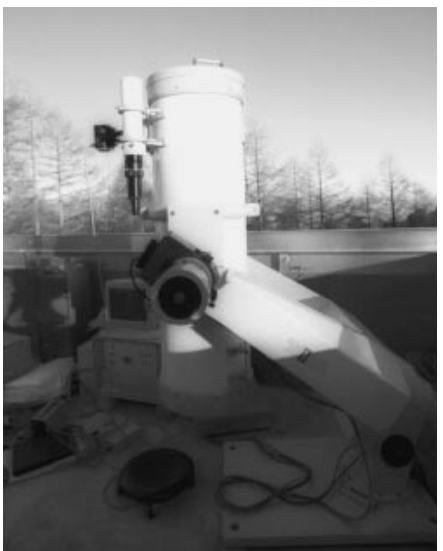

Fig. 3. Observational equipment at MNAO.

A $1 \mathrm{~K} \times 1 \mathrm{~K}$ back-illuminated type CCD camera is mounted on a $35-\mathrm{cm}$ optical telescope.

detect lines 30 to 40 times darker than can be normally detected with these cameras.

\section{Observations}

We performed a test observation of cataloged debris to evaluate the effectiveness of the line detection method. JAXA set up a 35-cm optical telescope (Takahashi $\varepsilon$ $350 \mathrm{~N}$ ), a fork-type equatorial mount (Showa 25EF), and a $1 \mathrm{~K} \times 1 \mathrm{~K}$ back-illuminated CCD camera (NIL FCC104B), at the Mt. Nyukasa Astronomical Observatory (MNAO) in November 2001. The observatory is in Fujimi Town in Nagano Prefecture, Japan. The focal length of the telescope is $1,248 \mathrm{~mm}$ and the field of view is $0.61 \times$ $0.61^{\circ}$. The CCD chip in the camera is a $1,024 \times 1,024$ pixel Marconi CCD47-20bi-AIMO, with a total area of $13.3 \times$ $13.3 \mathrm{~mm}$. The pixels are $13 \times 13 \mu \mathrm{m}$ each. Figure 3 shows the observational equipment. We observed the sky region at $\mathrm{RA}=9 \mathrm{~h} 19 \mathrm{~m}$ and $\mathrm{Dec}=+15^{\circ} 17$; taking 20 images each with a 3-minute exposure at dawn (04:21:51 to 05:23:17 JST) when the observation conditions for LEO debris are good. To suppress dark current causing electrical noise, the $\mathrm{CCD}$ chip was cooled to $-20^{\circ} \mathrm{C}$ using a thermo-electric cooler.

\section{Analysis and Results}

The 20 images were analyzed using the line detection algorithm described in Section 2. We used the IRAF (Image Reduction and Analysis Facility) ${ }^{13)}$ software (run under Linux) for the basic image processing, including rotation, addition, subtraction, multiplication and division of images. Perl scripts were used to direct IRAF to analyze the whole image almost automatically.

First, all the images were dark-frame subtracted and flatfielded. Then, fixed stars on each image were removed by subtracting a sequent image. Figures 4(a) and 4(b) show a raw image and the same image with fixed stars removed, respectively. The observation field is shifted slightly due to in- 


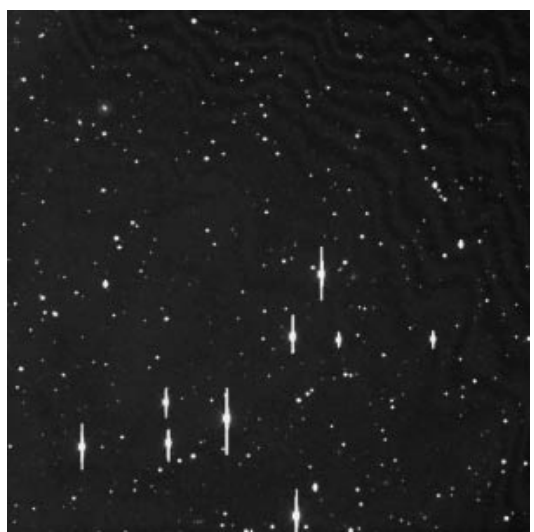

Fig. 4(a). Raw CCD image before fixed star removal.

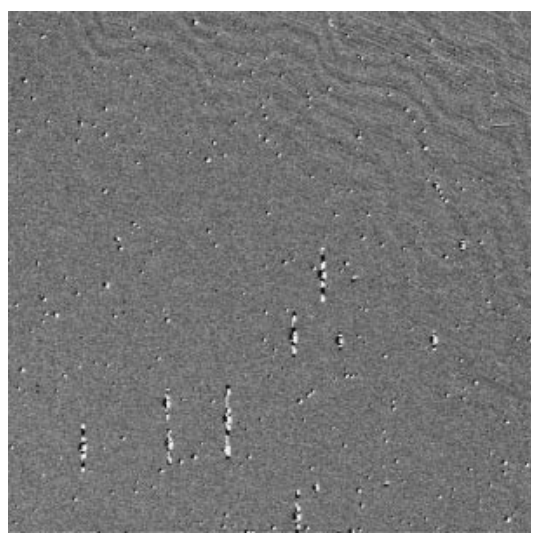

Fig. 4(b). Image with fixed stars removed by subtracting sequent image. Due to inaccurate setting of the telescope polar axis and changing atmospheric conditions, some effects of fixed stars still remain. In the top-right part of the image, there are fringe patterns characterizing back-illuminated CCD chips.

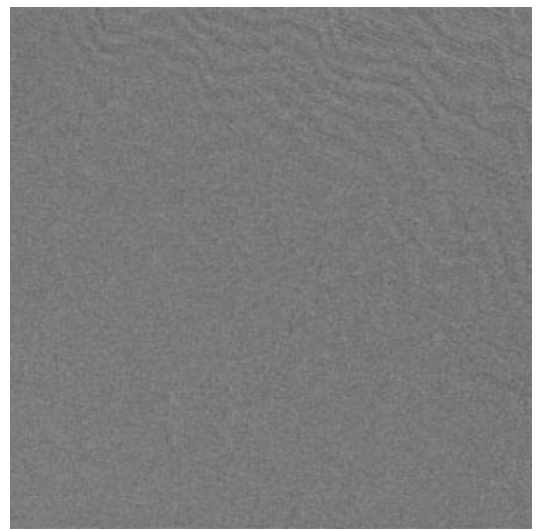

Fig. 4(c). Image with outlier pixels of brightness $3 \sigma_{\text {sky }}$ above and below median value of sky background adjusted to median value of sky background.

The effects in Fig. 4(b) are completely removed.

accurate setting of the telescope polar axis. Changing atmospheric conditions alter the apparent size of fixed stars. These factors cause the residual effects of fixed stars shown in Fig. 4(b). Although it is possible to exclude these effects by taking median values of all the pixels in each row, for detection of very weak lines, it is better to remove them before taking median values. Therefore, we investigated the standard deviation of the sky background values $\left(\sigma_{\text {sky }}\right)$ and then

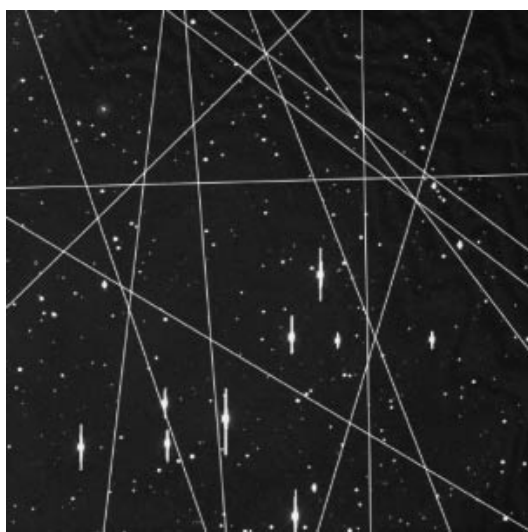

Fig. 5. Detected lines in observed image.

adjusted outlier values that were $3 \sigma_{\text {sky }}$ above or below the median value of the sky background to the median value of the sky background in order to remove such effects. Figure 4(c) shows the image after removing such effects.

Next, the 20 images were analyzed using the line detection software. Each image was rotated from $0^{\circ}$ to $180^{\circ}$ with in $0.5^{\circ}$ increments, and then the central $700 \times 700$ pixels was selected as the effective region. Then, the median values of all pixels in each row along the y-axis were calculated. Finally, the average and the standard deviation $\left(\sigma_{\text {median }}\right)$ of all the median values were calculated and the positions and rotation angles of the median values showing higher values than $5 \sigma_{\text {median }}$ were recorded. As a result of the analysis, we detected 12 lines. Table 1 and Fig. 5 give details of the detected lines and the positions where the lines passed through the observed area, respectively.

In this analysis, the software cut out an effective region of a $45^{\circ}$ rotation from all images in any rotation angle in order to simplify the analysis algorithm. However, when the rotation angle is around $0^{\circ}, 90^{\circ}$, or $180^{\circ}$, the effective region is almost equal to the raw image. The effective region should be determined for every rotation angle, because darker lines are more detectable as the effective region becomes wider. Equation (2) shows the pixel number of one side of the effective region at a proper rotation angle.

$$
N_{\text {effect }}=\frac{N}{\cos \phi+\sin \phi}
$$

Here, $N$ is the pixel number of one side of a raw image and $\phi$ is the rotation angle. In detecting a line that passes near the observation field center at a $45^{\circ}$ angle, the rotation and cutting out processes waste the region containing the line and consequently decrease the signal-to-noise ratio. An algorithm that considers such an effect is needed in order to detect the faintest lines possible.

\section{Identification of Detected Lines Using Catalog}

We investigated whether the detected lines had already been cataloged as space debris by comparing them with the catalog. First, the central celestial coordinates of the observed region were determined accurately. The pixel coordi- 
Table 1. Details of lines detected by line detection method.

\begin{tabular}{ccccc}
\hline No. of frame & Start time $($ UTC) & Rotation angle $\left(^{\circ}\right)$ & Coordinate (pixel) & Standard deviation $(\sigma)$ \\
\hline 6 & $19: 40: 17$ & 121.0 & 538 & 5.1 \\
9 & $19: 49: 31$ & 143.0 & 51 & 5.2 \\
10 & $19: 52: 35$ & 45.5 & 58 & 5.5 \\
11 & $19: 55: 40$ & 161.0 & 646 & 5.1 \\
11 & $19: 55: 40$ & 159.0 & 216 & 5.2 \\
12 & $19: 58: 46$ & 127.0 & 26 & 5.0 \\
14 & $20: 04: 55$ & 6.5 & 104 & 8.5 \\
14 & $20: 04: 55$ & 175.5 & 487 & 5.1 \\
14 & $20: 04: 55$ & 179.0 & 173 & 5.0 \\
15 & $20: 07: 59$ & 127.5 & 79 & 5.0 \\
17 & $20: 14: 08$ & 88.5 & 189 & 5.1 \\
\hline
\end{tabular}

Table 2. Details of cataloged objects that passed through field during observation time.

\begin{tabular}{|c|c|c|c|c|c|c|c|}
\hline SSC No. & International No. & Passage time (UTC) & Period (min) & Inclination $\left({ }^{\circ}\right)$ & Apogee $(\mathrm{km})$ & Perigee $(\mathrm{km})$ & $\operatorname{RCS}\left(\mathrm{m}^{2}\right)$ \\
\hline 08786 & 1974-089DN & $19: 45: 51.8-19: 45: 52.5$ & 115.8 & 102.3 & 1533 & 1450 & 0.0601 \\
\hline 17844 & $1986-101 \mathrm{~K}$ & $20: 05: 11.5-20: 05: 12.4$ & 102.9 & 82.5 & 907 & 882 & 0.0046 \\
\hline 19425 & 1976-126CC & $20: 12: 59.7-20: 13: 00.1$ & 113.4 & 66.3 & 1641 & 1120 & 0.0231 \\
\hline
\end{tabular}

nates of a number of bright fixed stars were calculated by the "daofind" command in IRAF, and the "ccxymatch" command determined the variables that convert pixel coordinates into celestial ones by comparing the pixel coordinates of the fixed stars with their celestial coordinates from the Guide Star Catalog ${ }^{14)}$ of the Hubble Space Telescope. The central celestial coordinates of the observed region calculated from the conversion variables were $\mathrm{RA}=9 \mathrm{~h} 19 \mathrm{~m}$ $52.79 \mathrm{~s}$ and $\mathrm{Dec}=+15^{\circ} 17^{\prime} 18.2^{\prime \prime}$.

Then, cataloged LEO satellites and debris passing through the observed region during the observation time were searched for using the STK (Satellite Tool Kit) ${ }^{15)}$ orbital calculation software manufactured by AGI, Inc. of the USA. The STK can search for objects passing through a specified field during a specified observation time by reference to orbital elements (in this case, TLE (Two Line Elements)) of a number of objects. It can also obtain more than 8,000 TLEs of satellites and debris offered by US Space Command via the Internet. We searched the objects that passed through our observed field from 04:21:51 to 05:23:17 (JST) on 23 November 2001 using 9221 TLEs updated on 20 November. The STK found three objects with SSC numbers 08786, 17844 and 19425. Table 2 shows the details of these three objects. We can identify the corresponding image number with the object by comparing the time when the image was taken with the calculated time when the object was in the field.

Table 1 and 2 show that the line of object with SSC number 17,844 (17,844 hereafter.) was detected in image No. 14. To confirm this, we compared the line geometry in the image defined by the TLE with the line defined by the rotation angle and the detected pixel coordinate. The celestial coor- dinates of the object at the start and end time in the field shown in Table 2 were calculated using the STK, and were then converted to the pixel coordinates on the image using the variables extracted previously. The angle of the straight line to the $\mathrm{Y}$-axis of the image, created by connecting these two points, was $6.51^{\circ}$. The rotation angle of the line detected by the line detection software was $6.5^{\circ}$. The software rotated the images with $0.5^{\circ}$ steps for analysis as a time-saving measure. Therefore, we re-analyzed image No. 14 with $0.1^{\circ}$ steps. The line on image No. 14 showed a peak at $6.3^{\circ}$. The results of the line detection for several rotation angles around $6.5^{\circ}$ are shown in Fig. 6. The $\mathrm{x}$ - and y-axes describe the pixel coordinates, perpendicular to the row along which the median values are calculated, and their values.

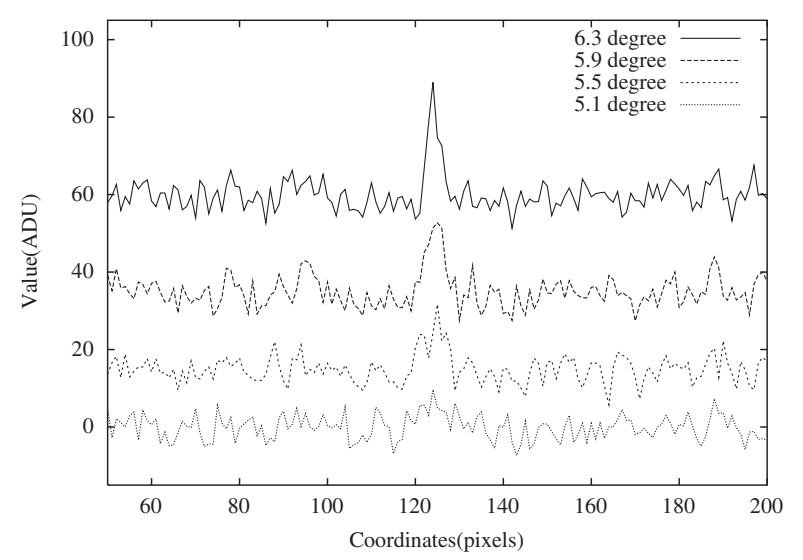

Fig. 6. Results of line detection at various rotation angles.

The solid, long-dashed, short-dashed and dotted lines show the results for rotation angles of $6.3^{\circ}, 5.9^{\circ}, 5.5^{\circ}$ and $5.1^{\circ}$, respectively. Appropriate constants were added to the upper three results for clear presentation. 


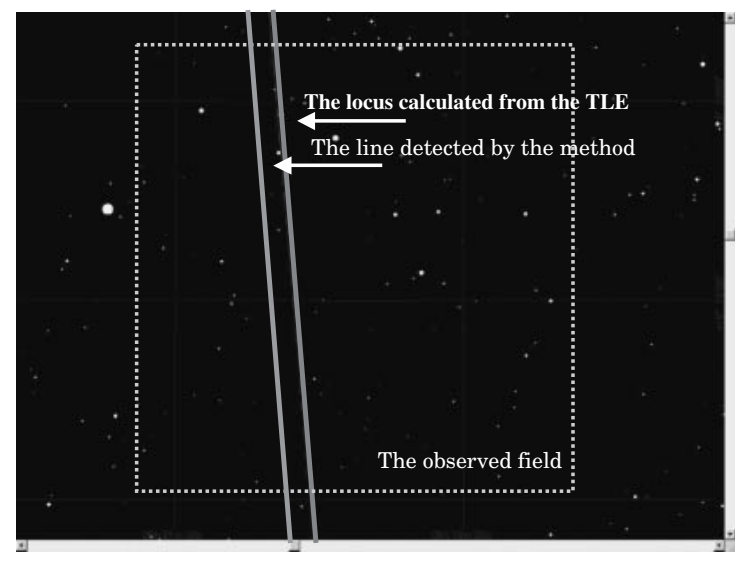

Fig. 7. Locus of 17,844 in observed field calculated from TLE, and position detected by line detection method.

The solid, long-dashed, short-dashed and dotted lines show the results for rotation angles of $6.3^{\circ}, 5.9^{\circ}, 5.5^{\circ}$ and $5.1^{\circ}$, respectively. Appropriate constants were added to the upper three results for clear presentation. Figure 6 indicates that the effect of the line becomes noticeable as the rotation angle approaches $6.3^{\circ}$. We calculated the distances of the two lines (the line calculated from the TLE and that detected line by the line detection software) from the image center. The difference in the distances was 82.1 pixels corresponding to just $0.0049^{\circ}$. These results show that the detected line on image No. 14 was the locus of 17,844 . The locus of the debris calculated from the TLE and the position of the detected line in the field are shown in Fig. 7.

The radar cross section (RCS) of this debris is $0.0046 \mathrm{~m}^{2}$ as described in Table 2. The RCS is the cross section of reflection estimated from the intensity of the reflection echo. Although this depends strongly on the material and the shape of the debris, it is possible to interpret RCS as an actual size to a first order approximation. The RCS of the debris shows a size of about $7 \mathrm{~cm}$. Two pieces of debris bits in addition to 17,844 should have been detected based on their apogees and perigees. However, the orbital determination of TLEs is not so accurate and our $0.61 \times 0.61^{\circ}$ field of observation (actually, the field used for the analysis is $0.42 \times 0.42^{\circ}$ ) is so narrow that these two pieces of debris might not have passed through our field. Since the line detection method can determine where objects passed through the sky with arc second accuracy (as in the case of 17,844), it could be used to improve orbital determination.

The objects in Table 1 other than 17,844 are thought to be non-cataloged debris or meteors. Since the method detects lines after taking CCD images, it is impossible to determine the velocities of detected objects, meaning we cannot determine the altitude of detected debris or distinguish debris from meteors. To investigate the environment of LEO debris in space, it is important to solve these problems. Double-site observation (Fig. 8) can solve the problems and multi-site observation can observe the debris environment at various altitudes simultaneously.

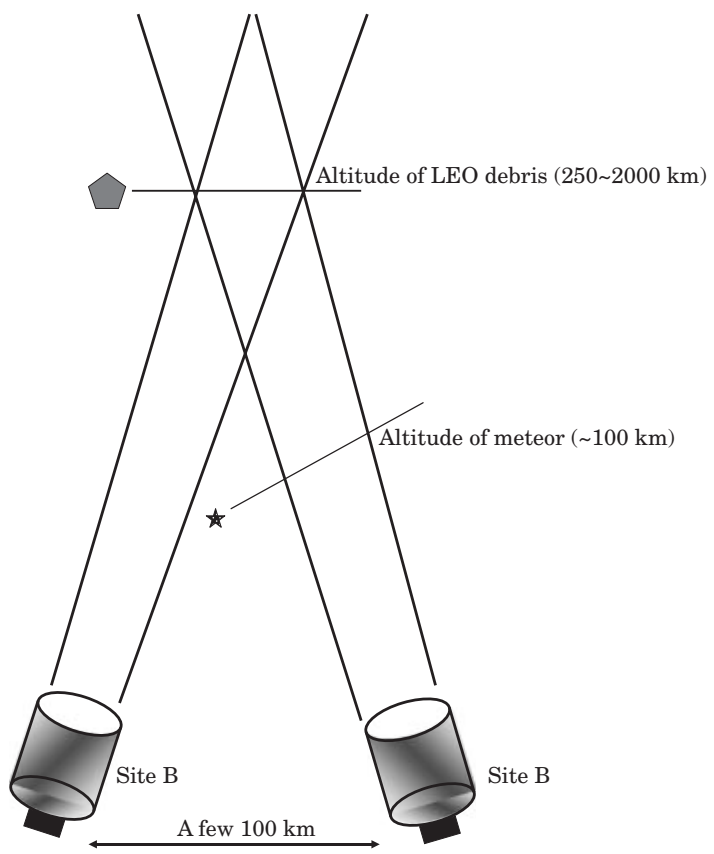

Fig. 8. Observation method to determine altitude of LEO debris. It is possible to remove lines created by meteors because meteors appear at a height of around $100 \mathrm{~km}$. LEO debris at various altitudes can be detected by changing telescope directions.

\section{Ability to Detect LEO Debris}

We have shown that a $35-\mathrm{cm}$ telescope and $1 \mathrm{~K} \times 1 \mathrm{~K}$ CCD camera can detect debris of a few $\mathrm{cm}$ using the line detection method. This section describes the power of the method using images taken by the equipment at MNAO (the $35-\mathrm{cm}$ telescope and $1 \mathrm{~K} \times 1 \mathrm{~K}$ CCD camera). We used the actual brightness of fixed stars and the actual sky background noise on the images in the investigation. We also investigated the power of the method using the equipment at the BSGC.

\subsection{Using equipment at MNAO}

First, we extracted the equation that transformed the brightness of fixed stars (magnitude) into digital values on CCD images (ADU value: Analog to Digital Unit) using one image of a 3-minute exposure and the Guide Star Cata$\log$ of the Hubble Space Telescope. The ADU values of 14 fixed stars on the image listed in the Catalog as stars of magnitude 13.5 to 15.1 were investigated. The average of the coefficients gave the relation between the magnitude and ADU value described as:

$$
F=10^{(27.698-M a g) / 2.5}
$$

Here, Mag and $F$ mean the magnitude of an object and its ADU value on the CCD image, respectively. The magnitudes of debris of various sizes at various altitudes are listed in Table 3. The albedo is 0.1 in this case.

We then calculated how long debris took to pass through the observation field for various altitudes of LEO. For simplicity, we assumed that the observed direction was the zenith and debris passed through $0.61^{\circ}$ parallel to one side of the observed field. We also ignored the effect of the Earth's 
Table 3. Brightness (magnitudes) of debris of various sizes at various altitudes. The albedo is assumed to be 0.1 .

\begin{tabular}{ccccc}
\hline \multirow{2}{*}{ Altitude $(\mathrm{km})$} & \multicolumn{4}{c}{ Size $(\mathrm{cm})$} \\
\cline { 2 - 5 } & 1 & 10 & 20 & 100 \\
\hline 2000 & 22.0 & 17.0 & 15.5 & 12.0 \\
1000 & 20.5 & 15.5 & 14.0 & 10.5 \\
500 & 19.0 & 14.0 & 12.5 & 9.0 \\
250 & 17.5 & 12.5 & 11.0 & 7.5 \\
\hline
\end{tabular}

rotation. The angular distance for the center of the earth that LEO debris passes through in the observed field depends on the altitude described as:

$$
\theta_{\mathrm{E}} \cong 2 \arctan \left\{\frac{h \tan (\theta / 2)}{h+6378}\right\}
$$

Here, $\theta$ and $\theta_{\mathrm{E}}$ are the angular distances that debris passes through in the image for the ground observer and the center of the earth, respectively. The unit is degrees $\left(^{\circ}\right) . h$ is the altitude and the earth is assumed to be a sphere with a radius of a $6,378 \mathrm{~km}$. The number of daily orbits of debris at altitude $h$ is shown as:

$$
n=\left(\frac{42164}{h+6378}\right)^{3 / 2}
$$

The time that it takes for debris at the altitude $h$ to pass through the angular distance $\theta$ is calculated as:

$$
t=\frac{240 \theta_{\mathrm{E}}}{n}=480 \arctan \left\{\frac{h \tan (\theta / 2)}{h+6378}\right\} /\left(\frac{42164}{h+6378}\right)^{3 / 2}
$$

Here, the unit is seconds. Equation (6) shows it takes 0.34, $0.69,1.45$ and 3.10 seconds for LEO debris at altitudes of $250,500,1,000$ and $2,000 \mathrm{~km}$, respectively, to pass through the observed field of $0.61^{\circ}$. If the pixel number along the locus of LEO debris on the image is $N$, the ADU value of one pixel is described as:

$$
P=\frac{t F}{180 N}
$$

As $F$ in Eq. (3) is the value for a 3-minute exposure, it must be scaled to the passage time of LEO debris. It also must be divided by the pixel number of one row as LEO debris passes through the image. The line detection method calculates the median of all pixel values of each row along the locus of LEO debris. The median values of each row are therefore described as in Fig. 2. If one median shows a sufficiently higher value than others, we can say that there is a line on the row. The ADU values of debris of various sizes at various altitudes are shown in Fig. 9. The solid, long-dashed, short-dashed and dotted lines show the results at altitudes of 250,500, 1,000 and 2,000 km, respectively.

The sky background noise (standard deviation of ADU value) on the image of a 3-minute exposure was 50 ADUs. Equation (1) shows that 1,024 pixels reduce the sky background noise to 1.875 ADU. The sky background noise is proportional to the square root of the incident photon number on the image if the readout noise is ignored. Thus, sky

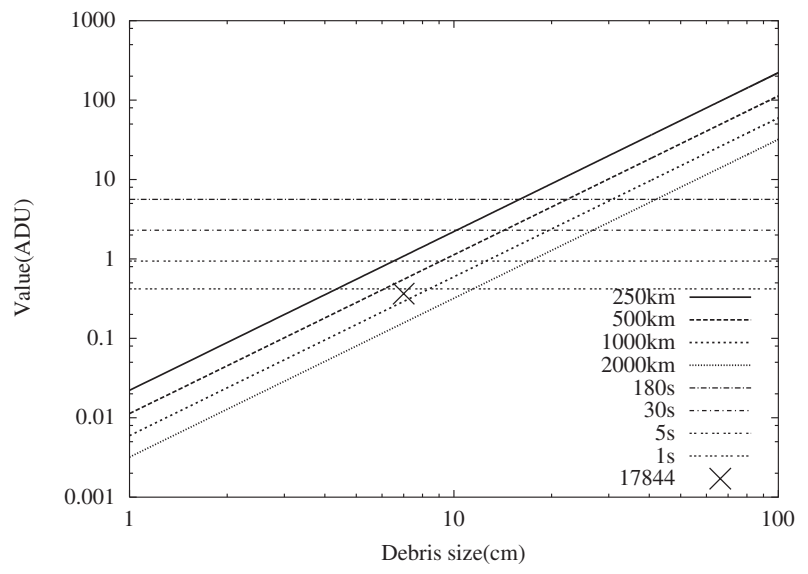

Fig. 9. ADU values of debris of various sizes at various altitudes for equipment at MNAO.

The solid, long-dashed, short-dashed and dotted lines show the values for altitudes of 250,500, 1,000 and 2,000 km, respectively. Triple the sky background values for various exposure times is also indicated by horizontal lines. The value of LEO debris 17,844 is marked with a cross.

background noise drops as exposure time becomes shorter. Figure 9 has lines to show triple the sky background values for various exposure times. Any LEO debris that shows a higher ADU value than the corresponding line for an exposure time may be detectable. However, the passage time of LEO debris depends on the altitude, as described in Eq. (6). To detect lines from LEO debris effectively, the exposure time must be longer than the passage time. Although the passage time calculated from the altitude (Eq. (6)) is the most effective exposure time to detect dark LEO debris, it would be very rare for LEO debris to pass through the field in just that time. The observation is also not efficient if the readout time of the $\mathrm{CCD}$ camera is longer than the exposure time, because the CCD cannot detect photons during readout and more than half the observation time is used for readout. We need to find an optimum exposure time, taking these factors in account.

Figure 9 shows that the equipment at MNAO can detect LEO debris smaller than $10 \mathrm{~cm}$ with a few seconds of exposure time. We calculated the passage time of 17,844 using its altitude of $900 \mathrm{~km}$ in Eq. (6), and the brightness of 7$\mathrm{cm}$ debris at an altitude of $900 \mathrm{~km}$ using Table 3. The ADU value of 17,844 when observed with the equipment at MNAO was calculated using these values in Eq. (7). The value is marked with a cross in Fig. 9. The exposure time of the image in which 17,844 was detected is $180 \mathrm{~s}$. Figure 9 shows that 17,844 is essentially undetectable because its ADU value is $10 \%$ of the detection limit (triple the noise level of a 180-second exposure). This occurs because the weak reflection produces an RCS smaller than the actual size and/or a much higher albedo than 0.1 of the highly reflective material of 17,844 . Spectroscopic observation is needed to determine the material of 17,844 .

\subsection{Using equipment at BSGC}

We also investigated the power of the line detection method using the equipment at BSGC in the same way as in Section 6.1. BSGC is used solely for observation of space debris 
and near-earth objects. ${ }^{16,17)}$ It has one 1-telescope and one 0.5-m telescope. Wide-field CCD cameras consisting of back-illuminated CCD chips are installed on both telescopes. BSGC started observations in April 2000. This investigation assumes that the $1-\mathrm{m}$ telescope and the $8 \mathrm{~K} \times$ $10 \mathrm{~K}$ CCD camera are used. The focal length of the telescope is $3,000 \mathrm{~mm}$ and the CCD chip is a back-illuminated ST-002A manufactured by SITe with a pixel size of $15 \times$ $15 \mu \mathrm{m}$ in a $2,048 \times 4,096$ array. The image areas of the 10 chips were aligned within $\pm 50 \mu \mathrm{m}$. Since the total number of pixels is $10,240 \times 8,192$, a line with a length of the average value of both sides $(9,216$ pixels $)$ is assumed to be created on the image. The 9,216 pixels correspond to $2.64^{\circ}$ in the sky when the focal length and pixel size are taken into account. Equations (4), (5) and (6) show that the passage times of LEO debris at altitudes of 250, 500, 1,000 and $2,000 \mathrm{~km}$ are $1.49,3.04,6.29$ and $13.39 \mathrm{~s}$, respectively for the equipment at BSGC. The field of view of one pixel (pixel scale) in the equipment at MNAO is 2.15 arc seconds. The corresponding value at BSGC is 1.03 arc seconds. Having the same pixel scale for both sites is convenient for comparison of sky backgrounds and ADU values of Eq. (7). We therefore assumed that $2 \times 2$ pixels of the camera at BSGC is equivalent to one pixel at MNAO, and the length of the line analyzed by the line detection algorithm is 4,608 pixels. The ratio of the telescope diameters shows that the equipment at BSGC can detect 8.2 times $(100 / 35 \times 100 / 35)$ as many photons as that at MNAO. This means that 8.2 times ADU values in Eq. (3) are gained by using the equipment at BSGC with a 3-minute exposure. Since the sky background noise is proportional to the square root of the number of entering photons, the sky background of a 3-minute exposure becomes $143(50 \times 100 / 35)$ ADUs. The ADU values for LEO debris of various sizes and at various altitudes were calculated using these values and Eq. (7) (Fig. 10). The three-times-ADU values of the sky background noise at various exposure times were also calculated. The solid, long-

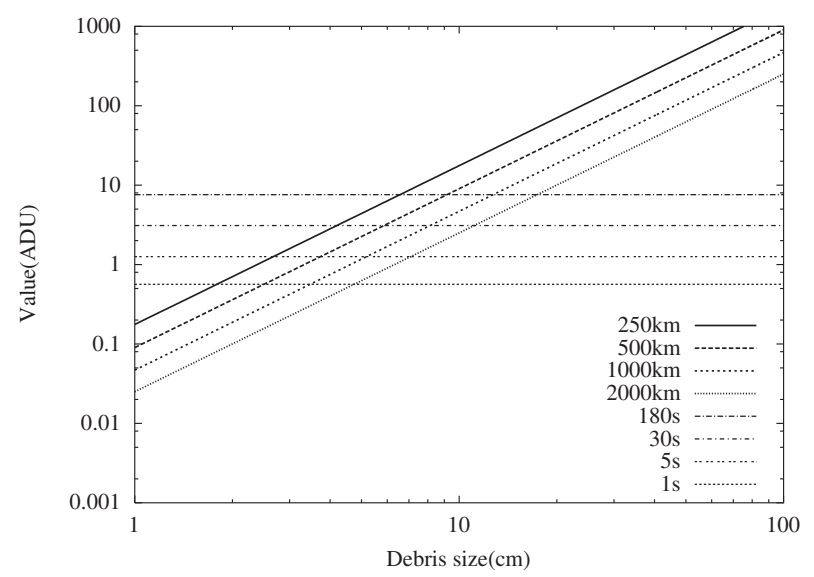

Fig. 10. ADU values of debris of various sizes at various altitudes for equipment at BSGC.

The solid, long-dashed, short-dashed and dotted lines show the values for altitudes of 250,500, 1,000 and 2,000 km, respectively. Triple the sky background values for various exposure times are also indicated by horizontal lines. dashed, short-dashed and dotted lines show the results for altitudes of 250,500, 1,000 and 2,000 km, respectively. They indicate that LEO debris of a few $\mathrm{cm}$ in size at an altitude of 300 to $500 \mathrm{~km}$ where the International Space Station is operating can be detected with an exposure time of a few seconds as shown in Fig. 10. Although the sky background at BSGC is about 1.3 times greater than that at MNAO as seen by comparing Figs. 10 and 9, the ADU value for LEO debris at BSGC is about 7.9 times greater than that at MNAO. As a result, the equipment at BSGC can detect LEO debris that is about 6 times darker than can be detected at MNAO.

\section{Conclusions}

We have proposed the line detection method for efficient detection of dark LEO debris using an optical telescope. It enables detection of a line on a CCD image caused by dark LEO debris that is invisible against the sky background, by assuming its direction on the image and accumulating pixel values along that direction. We succeeded in detecting LEO debris less than $10 \mathrm{~cm}$ in size at an altitude of $900 \mathrm{~km}$ by applying the method to images taken by equipment at MNAO.

LEO debris with a size of a few $\mathrm{cm}$ at altitudes of 300 to $500 \mathrm{~km}$ can be detected when this method is used with the equipment at BSGC.

However, this method cannot determine the velocity of LEO debris because analysis is performed after debris has passed through the image. This means that the method cannot distinguish LEO debris from meteors. The altitude of LEO debris is also unspecified. Double-site observation using two observatories about $100-\mathrm{km}$ apart can solve these problems.

The line detection method permits us to investigate the environment for LEO debris of less than $10 \mathrm{~cm}$ using optical telescopes, contributing greatly to solving the problem of space debris.

\section{References}

1) National Research Council: Orbital Debris - A Technical Assessment, Commission Engineering and Technical Systems, National Academy Press, Washington, D.C., 1995.

2) The Report on the Investigation about Optical Telescope for the Observation of Space Debris and NEO: Fundation for Promotion of Japanese Aerospace Technology (JAST) Press, Tokyo, 1997.

3) Taromaru, Y., Nonaka, K., Tajima, T., Sawabe, M., Yokota, T. and Isobe, S.: Overview of NASDA Orbital Analysis System and Bisei/ Kamisaibara Space Guard Centers, Proc. 23rd Int. Symp. Space Technology and Science, 2002, pp. 2330-2335.

4) Washio, T., Umehara, H. and Kimura, K.: An optical survey of geostationary satellites and debris, The Review of the Communications Research Laboratory, 45, 3/4 (1999), pp. 175-180.

5) Kawase, S.: Orbit Determination Accuracy for Optically Tracked near Synchronous Debris, J. Jpn. Soc. Aeronaut. Space Sci., 49 (2001), pp. 231-237.

6) Umehara, H. and Kimura, K.: An Optical Search for Near-synchronous Debris: Survey to 90 Degrees of Right Ascension, J. Jpn. Soc. Aeronaut. Space Sci., 49 (2001), pp. 1-8.

7) Nakajima, A., Yanagisawa, T., Kimura, T., Isobe, T., Tsuji, T., Yamamoto, M., Hoshino, T., Suzuki, M. and Futami, H.: Space Debris Observation by Ground-Based Optical Telescope, Proc. 22nd Int. Symp. 
Space Technology and Science, 2000, pp. 2055-2060.

8) Nakajima, A., Kimura, T., Yanagisawa, T. and Hoshino, T.: GEO/ LEO Space Debris Optical Observation Facilities in NAL, Proc. 23rd Int. Symp. Space Technology and Science, 2002, pp. 2336-2341.

9) Yanagisawa, T., Nakajima, A., Kimura, T., Isobe, T., Futami, H. and Suzuki, M.: Detection of Small GEO Debris by Use of the Stacking Method, Trans. Jpn. Soc. Aeronaut. Space Sci., 44 (2002), pp. 190199.

10) Yanagisawa, T., Nakajima, A. and Kimura, T.: The Stacking Method for Detection of Small GEO Debris and Moving Objects, Proc. 23rd Int. Symp. Space Technology and Science, 2002, pp. 2324-2329.

11) Yanagisawa, T., Nakajima, A. and Kimura, T.: Image Analysis for Detection of Small Objects, Proc. 46th Space Sciences and Technology Conference, 2002, pp. 57-62.

12) Pennycook, G.: Modification of the Boller and Chivens Telescope to f/6.25 and Photometry of IC5249's Halo, MS Thesis, Univ. Auckland, 1998; Report of the Work Done with the Grant-in-Aid for International Scientific Research in 1996-1998, Given by the Ministry of Education, Science, Sports and Culture of Japan, 2000, pp. 204-310.

13) http://iraf.noao.edu/iraf-homepage.html

14) http://www-gsss.stsci.edu/gsc/gsc.html

15) http://www.stk.com/

16) Isobe, S. and Japanese Spaceguard Association.: Japanese $0.5 \mathrm{~m}$ and $1.0 \mathrm{~m}$ Telescope to Detect Space Debris and Near-Earth Asteroids, $A d$ vances in Space Res., 23 (1999), pp. 33-36.

17) Isobe, S., Mulherin, J., Way, S., Downey, E., Nishimura, K., Doi, I. and Saotome, M.: A Cost Effective, Advanced-Technology Telescope System for Detecting Near Earth Objects and Space Debris, Proc. SPIE, In Telescope Structures, Enclosures, Assembly/Integration/ Validation, and Commissioning, 4004, 2000, pp. 382-388. 